\title{
Comparison of Gut Microbial Diversity in Beijing Oil and Arbor Acres Chickens
}

-Author(s)
Chen ZM'
Chang WH'
Zheng AJ'
Zhang S'
Cai HY'
Liu GH'
' The key laboratory of feed biotechnology of
the Ministry of Agricultural, Feed Research
Institute, Chinese Academy of Agricultural
Sciences, 12 Zhongguancun South Street,
Beijing, China

\section{Mail Address}

Corresponding author e-mail address G. H. Liu

The key laboratory of Feed Biotechnology of the Ministry of Agriculture, Feed Research Institute, Chinese Academy of Agricultural Sciences, No. 12 Zhongguancun South Street, Beijing 100081, China.

Tel: $\quad$ +861082106077

Email: liuguohua@caas.cn

\section{aKeywords}

Beijing oil chicken, Arbor Acres chicken gut microbial diversity, High-throughput sequencing.

\section{ABSTRACT}

The Beijing oil (BJO) chicken is an autochthona Chinese breed which shows outstanding meat quality characteristics compared with fast-growing imported chickens such as Arbor Acres (AA) chickens. Gut microbial diversity has been shown to influence host factors such as energy and nutrient metabolism, immune response and fertility. Consequently, it is hoped that analyses into host microbial populations will ultimately help to improve the quality of associated meat products. Two distinct chicken breeds, Arbor Acres (AA) and Beijing oil (BJO), were selected to analyze the composition of the 16S rRNA gene V3-V5 hypervariable regions using high-throughput sequencing technology. Upon elucidation of gut-colonizing bacteria, the Firmicutes were observed to encompass the dominant phylum. The abundance of Firmicutes in the BJO chickens (84.9\%) was lower than in the AA chickens (89.9\%). Conversely, the prevalence of Proteobacteria was $4.8 \%$ in BJO chickens, with a significantly lower abundance observed in $A A$ chickens (1.3\%). In the cecum, the Firmicutes were once more the dominant phylum in BJO chickens (60.2\%) and AA chickens (63.7\%). The abundance of bacteroidetes was $37.7 \%$ in BJO chickens and $34.9 \%$ in AA chickens, respectively. These discoveries provide a perception into the composition of the gut microbiotain both breeds. The study also provides a foundation for future research relating to gut bacterial factors that may influence the development and progression of gastrointestinal disease in chickens and other animals.

\section{INTRODUCTION}

The Beijing oil (BJO) chicken is a native Chinese breed and is regarded to show perfect meat quality compared with fast-growing imported chickens (Li et al., 2009; Lu et al., 2007; Zhang et al., 2007). Compared with the Arbor Acres (AA) chickens, the BJO chicken is extremely popular in China because of associated flavor characteristics. Previous studies have focused on the meat quality of the BJO chicken (Cherian et al., 2002; DeVol et al., 1988; Jiang et al., 2011; Matteo et al., 2007). To date, there has been a dearth of comparative metagenomic analyses pertaining to the role of gut microorganisms and microbial diversity in BJO and AA chickens. Gut microbiota constitute complex ecosystems, which display symbiotic relationships with associated hosts and play an important role in host performance. Host physiological, immunological, nutritional, and metabolic statuses are all affected by these interactions (Benson et al., 2010; Neish, 2009; O'Hara, 2006; Wardwell et al., 2011; Zhao et al., 2013). Previous research has shown that the host genotype is an important factor in dictating the composition of the gut microbiota 
(Khachatryan et al., 2008). Furthermore, evidence emerging from studies related to mice, chickens, and zebra fish show that the host influences the diversity and population composition of the gut microbiota (Rawls et al., 2006; Salzman et al., 2010; Vijay-Kuma et al., 2010). Moreover, a single gene difference in the host genome can affect the population structure of the gut microbiota. As mentioned above, the gut microbiota can influence animal health and studies that have investigated this phenomenon have already been conducted in chickens. However, relatively little is known regarding the difference between BJO and AA chickens in relation to their microbiota. Therefore, we selected 36 BJO chickens and 36 AA chickens to conduct a study into the composition of gut microbiota at 42 days (slaughter age). The objective of the present study was to compare gut microbiota between AA and BJO chickens.

\section{MATERIALS AND METHODS}

\section{Birds, Diets and Sample Collection}

Thirty-six BJO and thirty-six AA chickens were utilized as part of this experiment. Individuals from each breed had the same genetic background. Thestudy was initiated simultaneously for all birds and associated individuals were randomly distributed into six replicate groups for each breed; each group comprised six BJO birds and six AA birds. Birds were raised in an environmentally controlled room with four floor pens. Food and water were provided ad libitum during the experiment. Identical diets were employed for all individuals and the diets for the starter (1-21 d) and grower ( $\geq 22 \mathrm{~d}$ ) phases were formulated to meet, as much as possible, the recommendations for the two breeds (NRC, 1994; Ministry of Agriculture of P. R. China, 2004). Birds were slaughtered at typical market ages.

After 42 days, the chickens were slaughtered, and the contents of intestinal segments, including the ileum and cecum, were collected. Samples were snapfrozen in liquid nitrogen and stored at $-70^{\circ} \mathrm{C}$. Protocols used for this experiment were consistent with the Guidelines for the Care and Use of Laboratory Animals established by the Beijing Association for Laboratory Animal Science. The protocols were approved by the Animal Ethics Committee of the Feed Institute, Chinese Academy of Agricultural Sciences.

\section{$16 S$ r RNA gene amplification}

The V3-V5 region of the 16S r-RNA gene was PCR amplified using one pair of primer (forward primers: $5^{\prime}$
CCTACGGGAGGCAGCAG 3' and reverse primers: 5' CCGTCAATTCMTTTRAGT 3') with metagenomic DNA template and Master mix (Fermentas, UK). A total of 25 II of reaction mixture consisted of 40 pmol of each primer, 30-75 ng of template DNA, 12.5 II of Master mix (Fermentas, UK). The PCR amplification was performed by Thermal Cycler ( $A B I$, USA) and PCR conditions were adjusted in laboratory. The $P C R$ reactions were performed using the following conditions: $94^{\circ} \mathrm{C}$ for 4 min, followed by 27 cycles at $94^{\circ} \mathrm{C}$ for $30 \mathrm{~s}, 55^{\circ} \mathrm{C}$ for $45 \mathrm{~s}$, and $72^{\circ} \mathrm{C}$ for $60 \mathrm{~s}$; and a final extension step of $7 \mathrm{~min}$ at $72^{\circ} \mathrm{C}$. The PCR products that were generated were excised from a $1.5 \%$ agarose gel and purified using an Axy-Prep DNA Gel Extraction Kit (Axygen, AP-GX-500). The desired products were purified using Qiagen DNA Gel Extraction Kits (QIAGEN, CA) in accordance with the directions of the manufacturer. After quantification by nanodrop, the equimolar PCR products were pooled for each group.

\section{Pyrosequencing and sequence analysis}

Samples were subjected to pyrosequencing 454 Life Sciences technology based on high throughput sequencer (GSFLXRoche) with Titanium chemistry. Briefly, the sequencing library was prepared of $16 \mathrm{~S}$ rRNA gene amplicons by applying adapters on both ends of the fragments as well as MID as described by manufacturer. The resulting products were quantified using PicoGreen (Invitrogen) and a Qubit fluorometer (Invitrogen) before sequencing using Roche-454 GS FLX titanium chemistry. Emulsion PCR was carried out to clonally amplify fragments on sequencing beads, followed by its recovery and loading onto Pico titre plate along with enzyme beads. Pyrosequencing was carried out for 200 cycles with the flow of $A, T, G$ and $C$ nucleotides sequentially followed by image capture. Captured images were processed by image processing software to get sequencing reads. 16S rRNA gene sequence ( $>200$ bp) data were assigned to a bacterial taxonomic hierarchy using the ribosomal database project (RDP) Release 10 classifier. The classifier estimates the classification reliability by using bootstrapping. For bacterial taxonomic hierarchy a bootstrap cut off threshold of $95 \%$ was used.

\section{Diversity and Statistical Analysis}

Alpha diversity analysis which contained Chao1, ACE, Simpson, Shannon were performed using the 
summary single command of MOTHUR software (http://www.mothur.org/).

Statistical differences in basal characteristics between the groups were calculated by one-way analysis of variance. $p<0.05$ was considered statistically significant. All statistical analyses were performed using SPSS 16.0 software.

\section{RESULTS}

\section{Sequencing and Classification}

Total DNA was extracted from ileum and cecum samples of 72 chickens which were composed of $36 \mathrm{AA}$ and 36 BJO chickens, V3-V5 regions of $16 \mathrm{~S}$ rRNA gene were PCR amplified from each of those 72 DNA samples. $P C R$ products of $V 3-V 5$ regions were sequenced using 454 Life Sciences technology. After the quality control, a total of 877,392 and 677,268 validated sequences reads of V3-V5 16S rRNA sequences reads from the 72 ileum and cecum samples respectively with an average of 24372 and 18813 sequences reads for each sample were obtained in AA chickens. In BJO chickens, a total of 666,144 and 767,448 validated sequences reads of V3-V5 16S rRNA sequences reads from the 72 ileum and cecum samples respectively with an average of 18504 and 21318 sequences reads for each sample were obtained, which were classified into different Operational Taxonomic Units (OTUs) based on the identity level at $97 \%$.

\section{Analysis of Alpha Diversity}

Alpha diversity was estimated by four indices which contained Chao, ACE, Simpson, Shannon. The results showed that Chao and ACE were significantly higher in the BJO chickens than in the AA chickens. $(p<0.05)$. Good agreement was also observed between the Simpson's index and the Shannon's. The Simpson's index (0.60 vs 0.39 ) was lower in the BJO chickens than in the AA chickens, while the Shannon's index (0.86 vs 1.79) was higher in the BJO chickens than in the AA chickens; suggesting that the diversity of gut bacterial community for the BJO chickens was higher than that of the AA chickens. (Table 1).

\section{Analysis of Ileum and Cecum Microbial Populations}

Analysis of the species at the phyla level and the genus level, Total of 21 phyla and 164 genera were obtained. The microorganisms were mainly bacteria, accounting for $99.80 \%$ of all microorganisms. Of these 21 phyla, Firmicutes encompassed the dominant phylum in the ileum of $A A$ and BJO chickens. The abundance of Firmicutes in BJO chickens (84.9\%) was lower than that in AA chickens (89.9\%). Conversely, the relative abundance of Proteobacteria was $4.8 \%$ in BJO chickens, higher than AA chickens (1.3\%) (Graph 1a). Compared with AA chickens, BJO chickens harbor a more complex microbiota. There were seven phyla in the ileum of BJO chickens, while there were only three phyla in the ileum of AA chickens. In the cecum, Firmicutes also constituted the most dominant phylum in BJO chickens (60.2\%) and AA chickens (63.7\%). The abundance of bacteroidetes was $37.7 \%$ in $\mathrm{BJO}$ chickens and $34.7 \%$ in AA chickens, respectively (Graph 1b).

At the genus level we detected 164 genera. In ileum, the distribution proportion was greater than $2 \%$ in 5 genera, and of these 5 genera, the proportion of Lactobacillus (61.3 vs $72 \%$ ), Streptococcus (2.3 vs $3.8 \%$ ), and Enterococcus (1.1 vs $2.3 \%$ ) were higher in the AA chickens than in the BJO chickens. On the contrary, Lactococcus (4.1vs $3.1 \%$ ), the unnamed genera belong to Enterobacteriaceae (3.9 vs $3.1 \%$ ) and unnamed genera belong to Ruminococcaceae (3.7 vs $2.5 \%$ ) was lower in the AA chickens than in the BJO chickens (Graph 1c). In cecum, the distribution proportion was greater than $2 \%$ in 9 genera, and of these 9 genera, the proportion of Bacteroides (16 vs $6.1 \%$ ), unnamed genera belong to Rikenellaceae (21.9 vs $12.2 \%$ ), Faecalibacterium (10.5 vs $6.4 \%$ ) and were higher in the BJO chickens than in the AA chickens. On the contrary, Ruminococcus (3.8vs 5.3\%), the unnamed genera belong to Ruminococcaceae ( 8.9 vs $14.6 \%$ ) , unnamed genera belong to Barnesiellaceae (3.2 vs $12.9 \%)$, Oscillospira (2.1vs $2.5 \%$ ) was lower in the BJO chickens than in the AA chickens. (Graph 1d).

Table 1 - Alpha diversity indices

\begin{tabular}{lccc}
\hline Groups $(n=36)$ & chao & ACE & simpon \\
\hline AA chicken & $168.97 \pm 55.11$ & $140.67 \pm 51.16$ & $0.60 \pm 0.19 *$ \\
BJO chicken & $258.64 \pm 78.85^{*}$ & $279.69 \pm 65.46 * *$ & $0.39 \pm 0.17$ \\
\hline
\end{tabular}

The richness estimators (ACE and Chao), diversity indices (Shannon and Simpson) were calculated using the MOTHUR software. ${ }^{*}$ p 0.05 ; ${ }^{* *} p<0.01$ 
Chen ZM, Chang WH, Zheng AJ, Zhang S, Cai HY, Liu GH

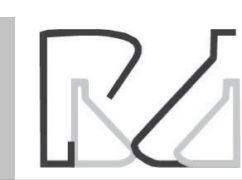

1 a

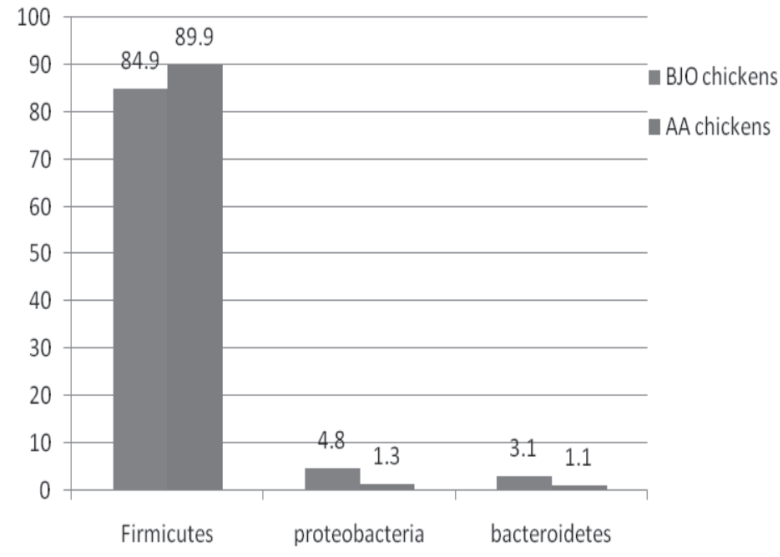

$1 c$
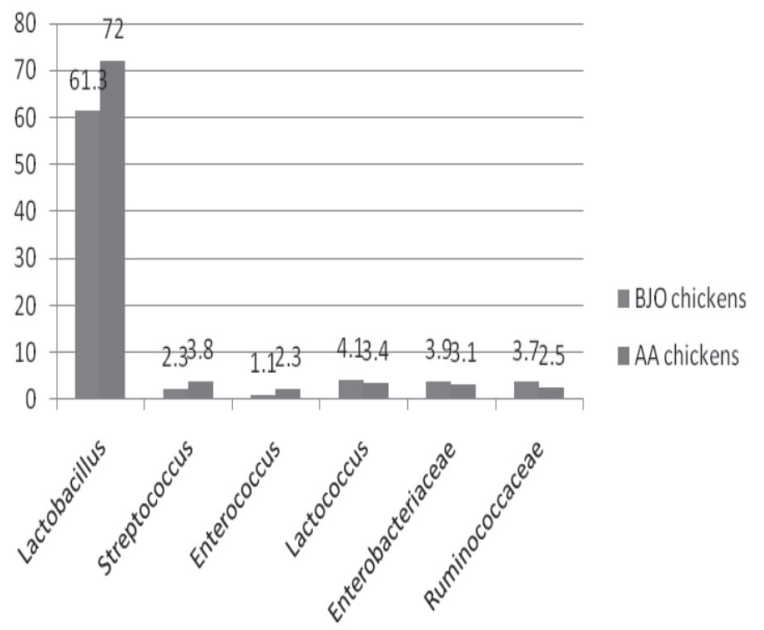

Graph 1 - Distribution of gut microbiota composition.

\section{Analysis of OTU Prevalence in Different} Species and Genders

To elucidate OTU prevalence in AA and BJO chickens, we generated an OTU venn map (Figure $1 \mathrm{a}, \mathrm{b})$. In the ileum, 636 and 789 OTUs were observed

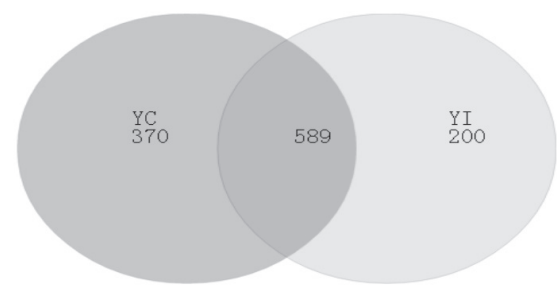

Figure 1a-OUT's amount in cecum and ileum of BJO

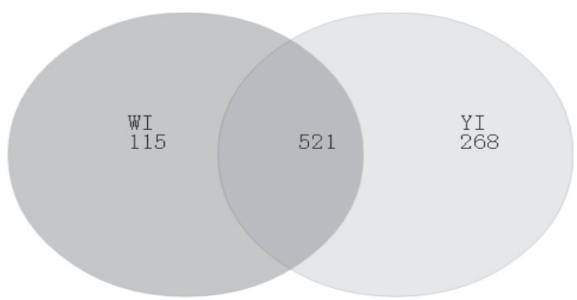

Figure 1c-OUT's amount in ileum of BJO and AA
Comparison of Gut Microbial Diversity in Beijing Oil and Arbor Acres Chickens

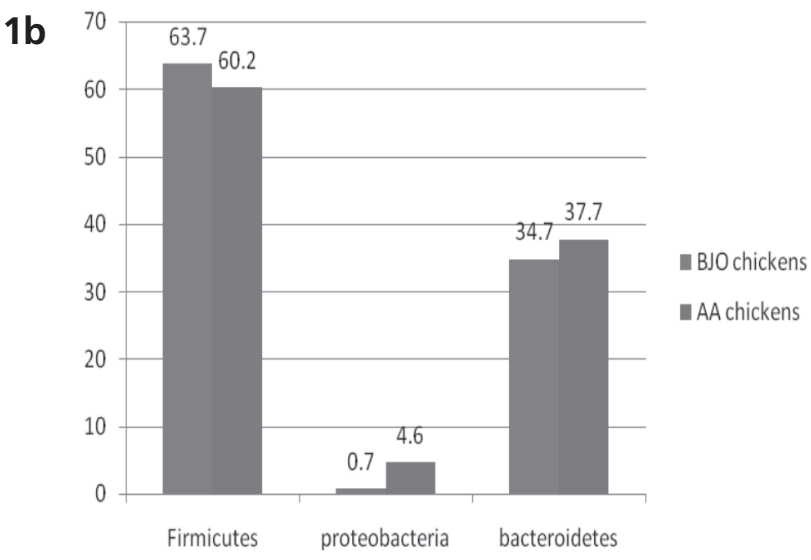

$1 d$
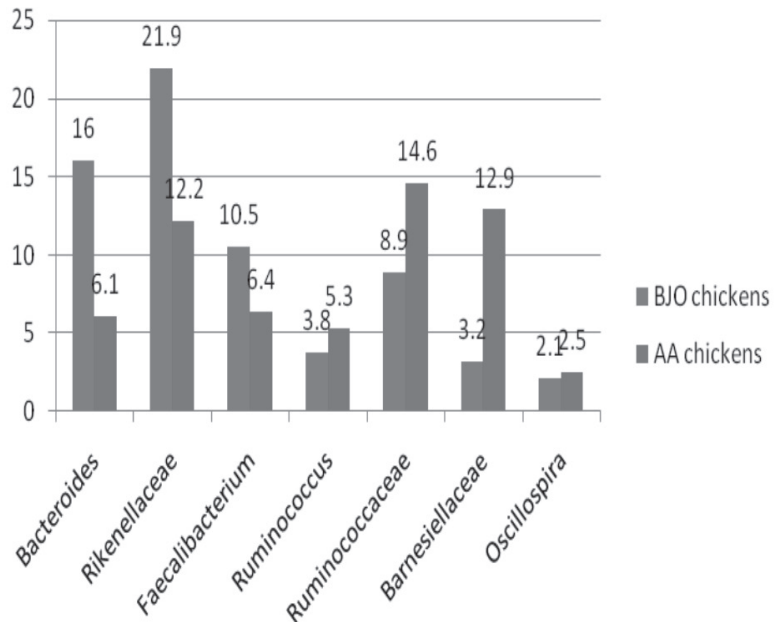

for AA and BJO chickens, respectively, and 521 of these OTUs appeared to overlap (Figure 1c). In the cecum, 1026 and 959 OTUs were observed in AA and BJO chickens, respectively, with 803 overlapping OTUs (Figure 1d).

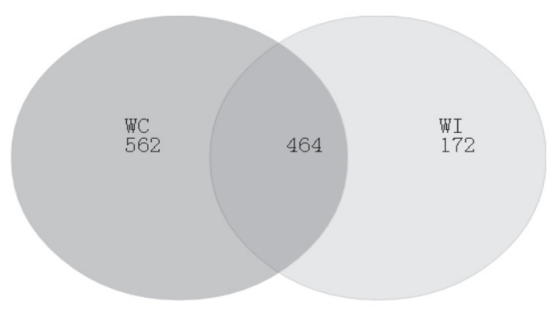

Figure $\mathbf{1 b}$ - OUT's amount in cecum and ileum of AA

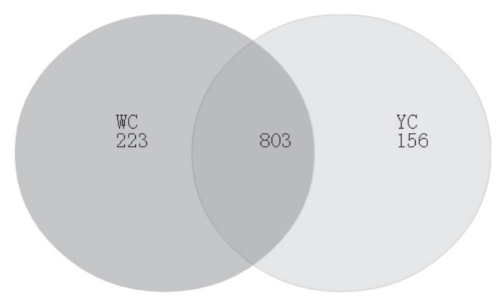

Figure 1d - OUT's amount in cecum of BJO and AA 
YC: OUTs in cecum of BJO chickens; YI: OUTs in ileum of BJO chickens; WC: OUTs in cecum of AA chickens;

WI: OUTs in ileum of AA chickens;

\section{Analysis of Species Abundance}

As part of this analysis, we detected 163 different genera. ANOVA results revealed that there were significant differences associated with the chicken genotypes following analysis $(p<0.05)$. To analyze genera differences in further detail, we generated multiple comparisons to show differences between the analyzed groups (Table 2 ).

Table 2 - ANOVA for Gene comparisons ( $p$ value)

\begin{tabular}{|c|c|c|}
\hline genus & $\begin{array}{c}\text { Ileum of BJO } \\
\text { and AA chickens }\end{array}$ & $\begin{array}{c}\text { cecum of BJO } \\
\text { and AA chickens }\end{array}$ \\
\hline Staphylococcus & $0.032^{*}$ & \\
\hline Odoribacter & & $0.0045^{* *}$ \\
\hline Lactobacillus & $0.0034 * *$ & $0.045^{*}$ \\
\hline Propionibacterium & $0.0139 *$ & \\
\hline Bacillus & $0.034^{*}$ & \\
\hline Clostridium & $0.02^{*}$ & \\
\hline Dorea & $0.037^{*}$ & \\
\hline Butyricicoccus & $0.049 *$ & \\
\hline Brevundimonas & $0.024^{*}$ & \\
\hline Ochrobactrum & $0.018^{*}$ & \\
\hline Achromobacter & $0.011^{*}$ & \\
\hline Delftia & $0.048^{*}$ & \\
\hline Enhydrobacter & $0.033^{*}$ & \\
\hline Pseudomonas & $0.042^{*}$ & \\
\hline Stenotrophomonas & $0.0005^{* *}$ & \\
\hline Blautia & $0.002 * *$ & $0.025^{*}$ \\
\hline Faecalibacterium & & $0.0066 * *$ \\
\hline Sutterella & & $0.026^{*}$ \\
\hline Bacillus & 0.001 * & \\
\hline Sphingomonas & $0.006^{* *}$ & \\
\hline Streptomyces & & $0.039 *$ \\
\hline Burkholderia & $0.008^{* *}$ & \\
\hline Acinetobacter & $0.013^{*}$ & \\
\hline Pseudomonas & $0.004 * *$ & \\
\hline Dehalobacterium & & $0.034^{*}$ \\
\hline Coprococcus & & $0.035^{*}$ \\
\hline Ruminococcus & $0.0086^{* *}$ & $0.017^{*}$ \\
\hline Holdemania & & $0.04^{*}$ \\
\hline Oscillospira & & $0.0097 * *$ \\
\hline
\end{tabular}

${ }^{*} p<0.05 ;{ }^{* *} p<0.01$

\section{DISCUSSION}

In this study, two distinct chicken breeds were analyzed. AA chickens are representative of fastgrowing chickens, while BJO chickens are indigenous breeds renowned for meat quality. This is the first study to report differences in the composition of BJO and
AA chicken gut microbiotas. The results characterize the gut microbiome of 36 healthy AA chickens and 36 healthy BJO chickens following high-throughput sequencing technology. Following analysis of ileumspecific samples, Firmicutes and proteobacteria were the two dominant phyla in both AA and BJO chickens. The abundance of Firmicutes in BJO chickens was lower than in AA chickens. Proteobacteria were relatively more abundant in BJO chickens compared with AA chickens. In the cecum, Firmicutes and bacteroidetes were the two most dominant phyla in AA and BJO chickens.

Firmicutes have been shown to be the most ubiquitous and common phylum in all vertebrates. Additionally, their presence correlates positively with the human ability to generate energy and absorb nutrients from feed components in rice (Jumpertz et al., 2011; Turnbaugh et al., 2008). Stanley et al. (2013) reported that an unknown class of firmicutes, which had been observed in the cecum of chickens, is negatively associated with apparent metabolizable energy (AME) and feed conversion ratio (FCR) performance as a consequence of high feed conversion ratios. The bacterial content associated with decomposing cellulose and starch is increased in the cecum of chickens exhibiting improved production performance (or a lower feed conversion ratio). The results of the analysis of the present study showed that the abundance of Firmicutes in BJO chickens was reduced compared with $A A$ chickens. This result is consistent with that observed by Stanley et al. (2013). The predominance of Firmicutes in AA chickens may be related to genetic differences between $A A$ and BJO chickens that have been exposed to the same diet and husbandry conditions. Genetic differences are likely to dictate the anatomical physiology and feeding habits of different species that ingest mainly insoluble fibers. These differences are also likely to be important in animals that use the cecum and large colon as the main sites for fermentation (Costa et al., 2012). Some researchers have also reported that a single gene difference in the host can significantly affect the population structure associated with the gut microbiota (Khachatryan et al., 2008; Salzman et al., 2010; Vijay-Kumar et al., 2010). Zhao et al. (2013) studied the population structure associated with the gut microbiota in two lines of chickens maintained using the same husbandry and dietary regimens. OTU of 190 microbiome species, 68 were affected by genotype (line), gender, and genotype-gender interactions. The host genotype may affect microbiota 
composition either directly through gut secretions, gut motility control and the modification of epithelial cell surfaces, or indirectly, through feed and lifestyle preferences. These results indicate that host genotypes affect gut microbiota.

Proteobacteria encompass the most physiologically diverse group of bacteria and these bacteria are well known for their utilization of a wide spectrum of carbon sources (Samanta et al., 2012). A study of gut microbiota in children found a greater abundance of Proteobacteria in European children who consumed a calorie-dense, high-fat, low-fiber diet compared with children from Burkina Faso who were low-fat, high fiber consumers (De Filippo et al., 2010). This difference revealed adaptive conditioning of the gut microbial community to the diet of African children, which could potentially improve their ability to harvest energy from indigestible polysaccharides. The high abundance of Proteobacteria in BJO chickens in this study is likely to contribute significantly to increasing digestion efficiency and assimilation. This phenomenon may play an important role in the generation of energy from nutrients. Further research is necessary to understand fully the relationship between specific functional molecules (enzymes) associated with microbial communities and BJO chicken metabolism. Bacterial species diversity is an important characteristic of a "healthy" gut microbiome. The data shows that BJO chickens demonstrate greater microbial community diversity and richness compared with AA chickens. This might be explained by differential rearing practices associated with BJO chickens, whereby BJO chickens were allowed to forage freely in both wild and captivity environments.

Bacteroidetes were detected the second most dominant gut flora genus observed in the cecum. The abundance of Bacteroidetes was observed to be $37.7 \%$ and $34.9 \%$ in BJO and AA chickens. This phylum can degrade high molecular weight compounds (including carbohydrates and proteins) in the intestine, thereby assisting the host in the acquirement of nutrients from the diet (Tremaroli and Backhed, 2012). Several studies have implicated that Bacteroidetes are capable of facilitating normal development of the gastrointestinal tract. Species associated with this genus are comprised of molecular effectors that facilitate the utilization of complex polysaccharides present in the colon. Additionally, the fermentative end products released by Bacteroidetes also provide nutrition and other beneficial properties to the host. It has been shown that gut Bacteroidetes generally produce butyrate, an end product of colonic fermentation, which is thought to have antineoplastic properties that contribute to the maintenance of a healthy gut (Ley et al., 2005). Bacteroidetes are also involved in bile acid metabolism and transformation of toxic or mutagenic compounds (Smith et al., 2006). These bacteria can adapt to distinct ecological niches due to the high plasticity of their genomes, which are capable of undergoing various genetic rearrangements, gene duplications, and lateral gene transfer reactions (Thomas et al., 2011). Therefore, this study postulates that the relative increased abundance of Bacteroidetes in BJO chickens is likely to contribute to host digestion adaptation. In humans, it is now well established that the ratio of Firmicutes and Bacteroidetes (F/B ratio) often correlates with body weight. The F/B ratio is significantly higher in obese individuals and significantly reduced during weight loss (Ley et al., 2005). However, the understanding of the extent to which host genotype and gender alter the gut microbiome of chickens is limited. Therefore, the study of the relationship between host genotypes, host gender, associated gut microbiomes, and native breed chicken domestication has become an area of growing scientific interest.

\section{ACKNOWLEDGMENTS}

This work was supported by the China Agricultural Research System (CARS-42-G15) and The Agricultural Science and Technology Innovation Program (ASTIP, 2014)

\section{REFERENCES}

Benson AK, Kelly SA, Legge R, Ma F, Low SJ, Kim J, et al. Individuality in gut microbiota composition is a complex polygenic trait shaped by multiple environmental and host genetic factors. Proceedings of National Academy Science USA 2010;107:18933-18938.

Cherian G, Selvaraj RK, Goeger MP, Stitt PA. Muscle fatty acid composition and thiobarbituric acid-reactive substances of broilers fed different cultivars of sorghum. Poultry Science 2002;81:1415-1420.

Costa MC, Arroyo LG, Allen-Vercoe E, Stampfli HR, Kim PK, Sturgeon A. Comparison of the fecal microbiota of healthy horses and horses with colitis by high throughput sequencing of the $\mathrm{V} 3-\mathrm{V} 5$ region of the $16 \mathrm{~S}$ rRNA gene. PLoSone 2012;7:1-11.

De Filippo C, Cavalieri D, Di Paola M, Ramazzotti M, Poullet JB, Massart S, et al. Impact of diet in shaping gut microbiota revealed by a comparative study in children from Europe and rural Africa. Proceedings of the National Academy Science USA 2010;107:14691-14696.

DeVol DL, Mckeith FK, Bechtel PJ, Novakofski J, Shanks RD, Carr TR Variation in composition and palatability traits and relationships between muscle characteristics and palatability in a random sample of pork carcasses. Journal of Animal Science 1988;66:385-395. 
Jiang RR, Zhao GP, Chen JL, Zheng MQ, Wen J. Effect of dietary supplemental nicotinic acid on growth performance carcass characteristics and meat quality in three genotypes of chicken. Journal of Animal Physiology and Animal Nutrition 2011;95:137-145.

Jumpertz R, Le DS, Turnbaugh PJ, Trinidad C, Bogardus C, Gordon Jl, Krakoff $J$. Energy-balance studies reveal associations between gut microbes caloric load and nutrient absorption in humans. American Journal of Clinical Nutrition 2011;94:58-65.

Khachatryan ZA, Ktsoyan ZA, Manukyan GP, Kelly D, Ghazaryan KA, Aminov RI. Predominant role of host genetics in controlling the composition of gut microbiota. PloS ONE 3:e3064.

Ley RE, Bäckhed F, Turnbaugh P, Lozupone CA, Knight RD, Gordon JR. Obesity alters gut microbial ecology. Proceedings of National Academy Science USA 2005;102:11070-11075.

Li WJ, Zhao GP, Chen JL, Zheng MQ, Wen J. Influence of dietary vitamin $\mathrm{E}$ supplementation on carcass and meat quality traits and gene expression related to lipid metabolism in chicken. British Poultry Science 2009;50:188-198.

Lu Q, Wen J, Zhang H. Effect of chronic heat exposure on fat deposition and meat quality in two genetic types of chicken. Poultry Science 2007;86:1059-1064.

Matteo B, Maria FC, Maria T, Rodriguez E, Giovanni L. Effect of feeding fat sources on the quality and composition of lipids of precooked ready-toeat fried chicken patties. Food Chemistry 2007;101:1327-1337.

Neish AS. Microbes in gastrointestinal health and disease. Gastroenterology 2009;136:65-80.

O'Hara AM, Shanahan F. The gut flora as a forgotten organ. EMBO Reports 2006;7:688-693.

Rawls JF, Mahowald MA, Ley RE, Gordon Jl. Reciprocal gutmicrobiota transplants from zebrafish and mice to germ-free recipients reveal host habitat selection. Cell 2006;127:423-433.

Salzman NH, Hung K, Haribhai D, Chu H, Karlsson-Sjöberg J. Amir E, et al. Enteric defensins are essential regulators of intestinal microbial ecology. Nature Immunology 2010;11:76-83.
Samanta AK, Torok VA, Percy NJ, Abimosleh SM, Howarth GS. Microbial fingerprinting detects unique bacterial communities in the faecal microbiota of rats with experimentally-induced colitis. The Journal of Microbiology 2012;50:218-225.

Smith CJ, Rocha ER, Paster BJ. The medically important Bacteroides spp in health and disease. The Prokaryotes 2006;7:381-427.

Stanley D, Geier MS, Denman SE, Haring VR, Crowley TM, Hughes RJ, Moore RJ. Identification of chicken intestinal microbiota correlated with the efficiency of energy extraction from feed. Veterinary Microbiology 2013;164:85-92.

Thomas F, Hehemann JH, Rebuffet E, Czjzek M, Michel G. Environmental and gut bacteroidetes: the food connection. Frontiers in Microbiology $2011 ; 2: 93$.

Tremaroli V, Bäckhed F. Functional interactions between the gut microbiota and host metabolism. Nature 2012;489:242-249.

Turnbaugh PJ, Bäckhed F, Fulton L, Gordon Jl. Diet-induced obesity is linked to marked but reversible alterations in the mouse distal gut microbiome. Cell Host \& Microbe 2008;3:213-223.

Vijay-Kumar M, Aitken JF, Carvalho A, Cullender TC, Mwangi S, Srinivasan $\mathrm{V}$, et al. Metabolic syndrome and altered gut microbiota in mice lacking Toll-like receptor 5. Science 2010;328:228-231.

Wardwell LH, Huttenhower C, Garrett WS. Current concepts of the intestinal microbiota and the pathogenesis of infection. Current Infectious Diseases Report 2011;13:28-34.

Zhang GM, Wen J, Chen JL, Zhao GP, Zheng MQ, Li WJ. Effect of conjugated linoleic acid on growth performances carcase composition plasma lipoprotein lipase activity and meat traits of chickens. British Poultry Science 2007;48:217-223.

Zhao L, Wang G, Siegel P, He C, Wang H, Zhao W, et al. Quantitative genetic background of the host influences gut microbiomes in chickens. Scientific Reports 2013;3:1163. 
